\title{
晶硅炉热场用碳材料在硅蒸汽中的腐蚀行为研究
}

\author{
施 伟 ${ }^{1,2}$, 谭 毅 ${ }^{1,2}$, 郝建洁 ${ }^{1,2}$, 李佳艳 ${ }^{1,2}$ \\ (1. 大连理工大学 材料科学与工程学院, 大连 116024; 2. 辽宁省太阳能光伏系统重点实验室, 大连 116024)
}

摘 要: 对晶硅炉热场用三种碳材料(石墨、 $\mathrm{C} / \mathrm{C}$ 复合材料、硬质碳䍄)在不同腐蚀条件下进行了硅蒸汽腐蚀实验，研 究了不同碳材料的硅化腐蚀行为。结果表明: 三种材料的结构组成不同, 导致三种碳材料在高温环境下与硅蒸汽发 生硅化腐蚀的程度不同，硅蒸汽主要通过材料的孔洞以及缺陷扩散进入材料内部进行腐蚀，腐蚀造成材料表面产 生大量的裂纹, 并且会造成 $\mathrm{C} / \mathrm{C}$ 复合材料以及硬质碳䏨材料内部严重的破坏。材料中碳纤维的存在方式以及纤维 强度对硅化腐蚀程度有较大影响, $\mathrm{C} / \mathrm{C}$ 复合材料中的腐蚀主要是碳基体和碳纤维的剥离以及碳纤维的缩颈、繴裂等; 而由强度较差的短碳纤维为主要组成的硬质碳占材料的腐蚀最严重, 其内部碳纤维出现了明显的碎断、粉化。腐蚀 参数对材料硅化腐蚀的程度具有较大影响, 随着腐蚀温度的升高以及腐蚀时间的延长, 三种碳材料的硅化腐蚀程 度均增加。

关 键 词: 碳材料; 硅化; 腐蚀行为; 腐蚀参数

中图分类号: TB332 文献标识码: A

\section{Corrosion Behavior of Carbon Materials Used in Crystalline Silicon Furnace in Silicon Vapor}

\begin{abstract}
SHI Wei ${ }^{1,2}$, TAN Yi ${ }^{1,2}$, HAO Jian-Jie ${ }^{1,2}$, LI Jia-Yan ${ }^{1,2}$
(1. School of Materials Science and Engineering, Dalian University of Technology, Dalian 116024, China; 2. Key Laboratory for Solar Energy Photovoltaic System of Liaoning Province, Dalian University of Technology, Dalian 116024, China)

Abstract: The experimental of silicon vapor corrosion under different corrosion conditions were carried out on different carbon materials (graphite, $\mathrm{C} / \mathrm{C}$, rigid carbon fiber felt (RCFF)), which were used as thermal field materials in silicon crystal growth furnace. The silicification corrosion behavior of the three carbon materials was studied. The results showed that the three carbon materials are easily corroded by silicon vapor at elevated temperature. The composition and microstructure of the three carbon materials have great influence on silicification corrosion behavior. Silicon vapor can infiltrate into interior of the carbon materials by pores and flaws, and then react with substrates to cause corrosion. After silicification corrosion, many cracks are observed on the surface of the three carbon materials, and the carbon fibers are severely destroyed in C/C composites and RCFF. Silicification corrosion level is affected by the form and strength of carbon fibers in $\mathrm{C} / \mathrm{C}$ composites and RCFF. The corrosion of carbon fiber is necking and cleavage cracking for carbon fiber woven with high strength in $\mathrm{C} / \mathrm{C}$ composites, while that is fragmenting and pulverizing for short carbon fiber with poor strength in RCFF. The corrosion parameters also have significant influence on degree of
\end{abstract}

收稿日期：2016-09-14; 收到修改稿日期：2016-10-24

基金项目: 高等学校博士学科点专项科研基金(20130041110004); 国家自然科学基金重点项目(51574057); 中央高校基本 科研业务费专项基金(DUT15QY07)

Specialized Research Fund for the Doctoral Program of Higher Education(20130041110004); National Natural Science Foundation of China (51574057); Fundamental Research Funds for the Central Universities (DUT15QY07)

作者简介: 施 伟(1986-), 男, 博士研究生. E-mail: wshi0703@163.com

通讯作者: 李佳艳, 副教授. E-mail: lijiayan@dlut.edu.cn 
silicification corrosion. With the corrosion temperature increasing or corrosion time extending, the silicification corrosion degree for these carbon materials are all increased.

Key words: carbon materials; silicification; corrosion behavior; corrosion parameters

随着晶体硅材料在光伏产业中的广泛应用, 其 主要的生产设备一晶硅炉(单晶硅拉晶炉以及多晶硅 铸锭炉)也得到迅速发展。在晶体硅的生长过程中, 为了保证晶体的稳定生长, 通常需要控制热场, 因 此晶硅炉热场的稳定性显得尤为重要。晶硅炉的热 场材料包括: 石墨(Graphite)、炭/炭 $(\mathrm{C} / \mathrm{C})$ 复合材料以 及硬质碳毡(rigid carbon fiber felt, 简称: RCFF)等材 料, 其中石墨主要用于加热器、坩埚、防漏槽等, $\mathrm{C} / \mathrm{C}$ 复合材料主要用于导流筒、盖板、螺栓螺母等, 硬 质碳毡主要用于隔热保温层 ${ }^{[1-5]}$ 。

在晶体硅材料的实际生产过程中, 由于温度较 高, 用来盛放硅料的石英坩埚以及熔化的硅料均会 挥发产生大量的 $\mathrm{SiO}_{2}$ 和 $\mathrm{Si}$ 蒸汽, 这些气体与碳基的 热场材料接触时容易发生氧化反应和硅化反应而产 生腐蚀 ${ }^{[6-7]}$, 这种腐蚀会对材料造成严重的损害, 从 而影响热场的稳定性，同时对设备造成安全隐患。 因此, 研究晶硅炉用热场碳材料的腐蚀行为具有重 要的现实意义。

目前, 国内外研究学者对碳材料的腐蚀研究主 要集中在氧化腐蚀方面, $\mathrm{Zhou}^{[8]}$ 和陈志军 ${ }^{[9]}$ 等分别 研究了石墨和碳/碳复合材料的氧化行为, 徐伟 ${ }^{[10]}$ 和廖继乔 ${ }^{[11]}$ 等分别研究了碳材料的微观结构对氧 化性能的影响, 而对于硅化腐蚀的研究报道的文献 尚少, 曹伟伟等 ${ }^{[6]}$ 研究了石墨及炭/炭复合材料表面 的液相硅的腐蚀行为。然而, 在材料的实际使用过 程中, 更多的腐蚀行为主要是由气相的硅蒸汽与碳 材料的反应造成。因此, 本工作对晶硅炉热场用的 碳材料进行气相硅化腐蚀, 研究碳材料在硅蒸汽中 的腐蚀行为, 以期为晶硅炉用热场材料的优化以及 防护奠定基础。

\section{1 实验方法}

实验所用三种碳材料均由上海晶驰碳素有限公 司提供, 其具体参数指标见表 1 。碳材料的表面宏观 及微观形貌如图 1 所示, 其中石墨材料主要由石墨 颗粒高温等静压烧结制得, 其结构较为致密, 表面 存在少量的孔洞; $\mathrm{C} / \mathrm{C}$ 复合材料由碳纤维和热解碳 组成, 其中碳纤维束交错编织, 且由于生产过程中 碳纤维之间的缝隙无法完全被热解碳填充, 使得材
表 1 三种碳材料的参数指标

Table 1 Properties of three carbon materials

\begin{tabular}{cccc}
\hline $\begin{array}{c}\text { Carbon } \\
\text { materials }\end{array}$ & $\begin{array}{c}\text { Density } \\
/\left(\mathrm{g} \cdot \mathrm{cm}^{-3}\right)\end{array}$ & $\begin{array}{c}\text { Flexural } \\
\text { strength } \\
/ \mathrm{MPa}\end{array}$ & $\begin{array}{c}\text { Compressive } \\
\text { strength } \\
/ \mathrm{MPa}\end{array}$ \\
\hline Graphite & 1.80 & 39.2 & - \\
$\mathrm{C} / \mathrm{C}$ & 1.40 & 78.3 & - \\
$\mathrm{RCFF}$ & 0.18 & 1.5 & $0.35^{*}$ \\
\hline *. Compressive strength is the stress at $5 \%$ compression
\end{tabular}

*: Compressive strength is the stress at $5 \%$ compression deformation

料内部含有较多的孔隙; 硬质碳毡主要是由短碳纤维 的无序排列构成, 热解碳含量较少, 其对碳纤维的固 定较差，表面可以看到杂乱的纤维。该材料密度较低, 其孔隙较大且孔隙率极高, 达到 $90 \%$ 以上。

由热力学计算可知 ${ }^{[12]}$, 碳材料在室温 $1600^{\circ} \mathrm{C}$ 范围均可与硅发生反应造成腐蚀, 结合实际的晶硅 炉使用工况条件，设定不同的腐蚀温度(1400、 $\left.1500 、 1600^{\circ} \mathrm{C}\right)$ 和腐蚀时间 $(1 、 5 、 10 \mathrm{~h})$ 来研究腐蚀 参数对腐蚀行为的影响。实验步骤如下: 首先将硅 料置于石墨坩埚中，将尺寸为 $35 \mathrm{~mm} \times 35 \mathrm{~mm} \times$ $10 \mathrm{~mm}$ 的三种碳材料分别置于石墨坩埚上，盖住石 墨坩埚, 使硅蒸汽单侧接触碳材料, 然后将坩埚置 于真空碳管炉中, 以 $10^{\circ} \mathrm{C} / \mathrm{min}$ 的速率升温至设定的 实验温度保温, 整个实验过程采用高纯氩气保护。 随着温度升高, 硅料熔化后会产生大量的硅蒸汽, 扩散至碳材料表面即发生硅化反应。

采用 ZEISS SUPRA 55 型场发射扫描电子显微 镜对材料的微观形貌进行观察; 采用 Oxford 型能谱 仪分析腐蚀区域截面元素的分布情况，确定腐蚀的 深度; 采用 Bruker Ds Focus 型 X 射线衍射仪确定碳 材料表面硅化腐蚀区域的相结构。

\section{2 结果与讨论}

\section{1 碳材料硅化腐蚀的典型形貌特征}

图 2 为三种碳材料在 $1500^{\circ} \mathrm{C}$ 下硅化腐蚀 $5 \mathrm{~h}$ 后 的宏观形貌, 由图可以看出, 三种碳材料在硅蒸汽 腐蚀后表面均产生浅白色物质, 石墨和 $\mathrm{C} / \mathrm{C}$ 复合材 料表面的浅白色物质较为致密, 经 XRD 测试(测定 $\mathrm{C} / \mathrm{C}$ 复合材料表面, 如图 3(a)所示)发现浅白色物质 为 $\operatorname{SiC}(J C P D S$ 29-1129), 其最强衍射峰对应(111)面 


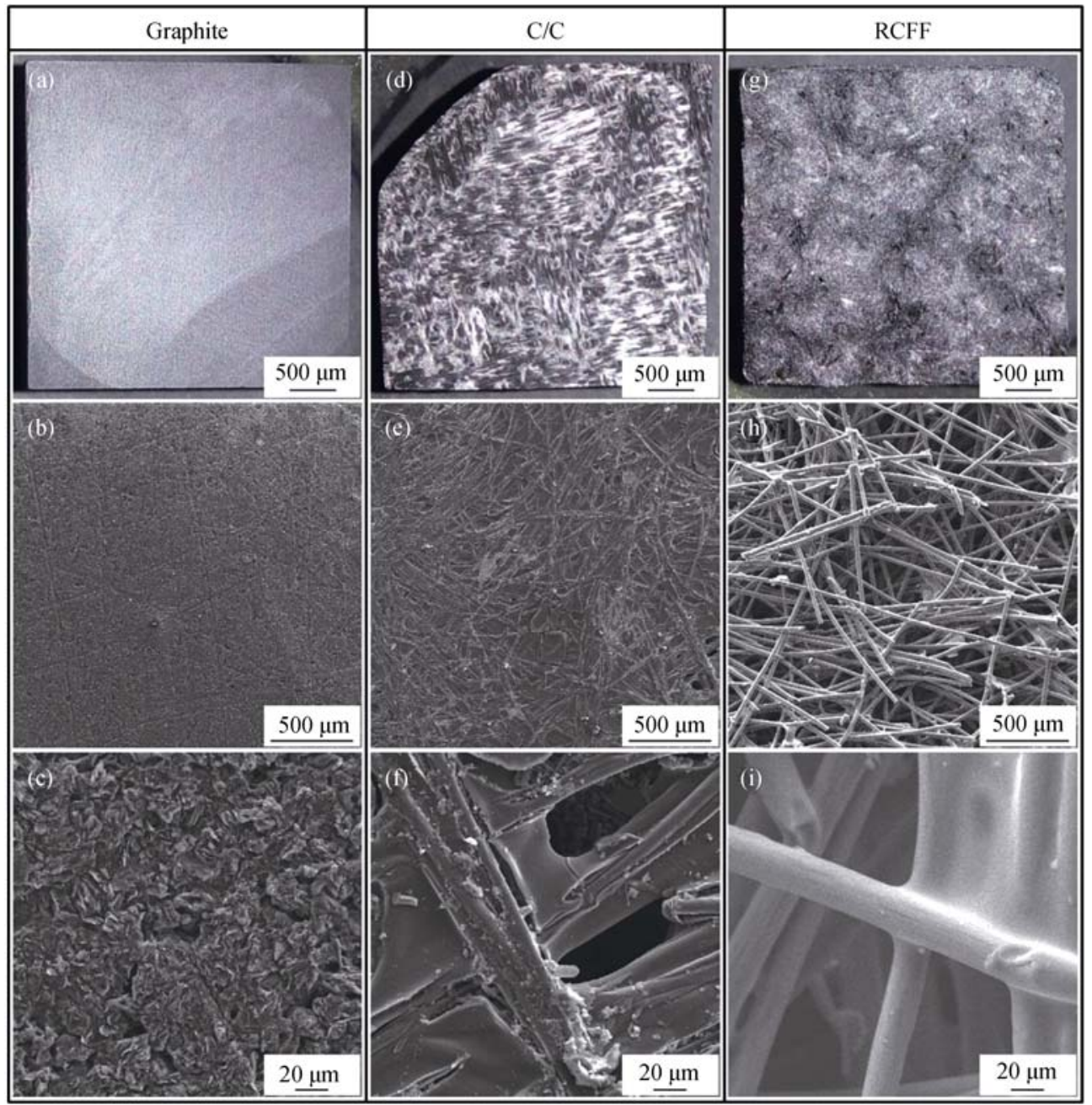

图 1 三种碳材料的表面宏观及微观形貌

Fig. 1 Macro- and micro-morphologies of three carbon materials (a)-(c) Graphite; (d)-(f) C/C composite; (g)-(i) Rigid carbon fiber felt
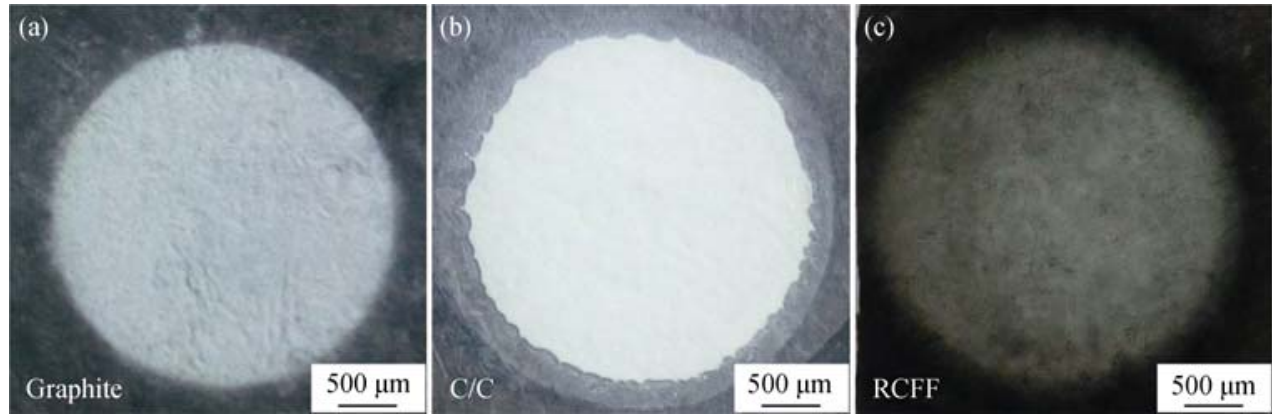

图 2 三种碳材料在腐蚀后的表面宏观形貌

Fig. 2 Macro morphologies of three carbon materials after corrosion

的衍射峰，(111)衍射峰前面的小衍射峰是由堆垛层 错引起的 ${ }^{[13]}$ 。图 3(b)为浅白色区域的微观形貌图, 从图 3(b)局部放大图(右上角)可以看到, 白色区域 是由直径极为细小的纳米线组成, 即碳材料经过硅 蒸汽腐蚀后，其表面形成了一层较薄的 $\mathrm{SiC}$ 纳米线。

$\mathrm{SiC}$ 纳米线的形成主要原因是：高温条件下, 极微量的氧与 $\mathrm{C}$ 和 $\mathrm{Si}$ 反应分别生成 $\mathrm{CO}$ 和 $\mathrm{SiO}$, 碳
材料表面固态的碳与 $\mathrm{Si}$ 或 $\mathrm{SiO}$ 气体反应在表面生成 $\mathrm{SiC}$ 晶核, 之后在 $\mathrm{SiC}$ 晶核上 $\mathrm{SiO}$ 和 $\mathrm{CO}$ 发生气一固 反应(Vapor-Solid:VS) ${ }^{[14]}$, 沿一维方向不断生长形成 $\mathrm{SiC}$ 纳米线。由于体系中的氧含量较少, 因此在碳材 料表面形成较薄的 $\mathrm{SiC}$ 纳米线层后，主要发生的是 $\mathrm{Si}$ 蒸汽与固态碳的腐蚀反应，反应方程式为: $\mathrm{C}(\mathrm{s})+$ $\mathrm{Si}(\mathrm{g}) \rightarrow \mathrm{SiC}(\mathrm{s})$ 。 

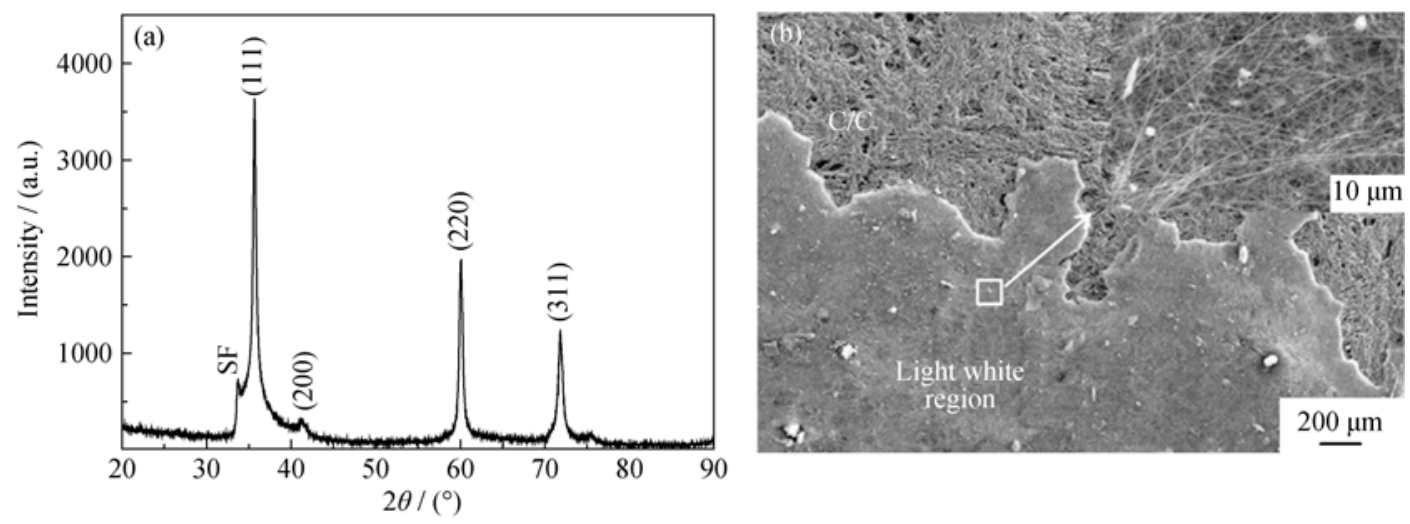

图 $3 \mathrm{C} / \mathrm{C}$ 复合材料表面 $\mathrm{SiC}$ 纳米线的 XRD 图谱以及微观形貌

Fig. 3 XRD pattern and microstructure of $\mathrm{SiC}$ nanowires on the surface of $\mathrm{C} / \mathrm{C}$ composites

白色的 $\mathrm{SiC}$ 纳米线层与基底的结合较弱, 经超 声清洗后极易脱落, 露出淡绿色的基底。图 4 为经 超声清洗后的三种碳材料微观形貌。从图 4(a) (d) 可以看到, 石墨材料经硅化腐蚀后, 表面变得粗糙, 且出现较多的微小裂纹(裂纹尺寸约为 $1 \sim 2 \mu \mathrm{m}$ ), 石 墨颗粒的表面被细小的 $\mathrm{SiC}$ 颗粒覆盖, 并且有少量 纳米线附着; 这主要是由于硅蒸汽与石墨颗粒反应 生成的 $\mathrm{SiC}$ 与基底石墨的热膨胀系数有差异(石墨热 膨胀系数 $5.8 \times 10^{-6} \mathrm{~K}^{-1}$, SiC 热膨胀系数 $4.5 \times 10^{-6} \mathrm{~K}^{-1}$ ), 从而会在材料内部产生热应力, 当热应力超过材料 自身强度时便会造成开裂, 裂纹为后续硅蒸汽的渗 入提供了通道, 又会进一步加速材料的腐蚀。从图 $4(\mathrm{e} \sim \mathrm{h})$ 可以看出, $\mathrm{C} / \mathrm{C}$ 复合材料表面整体变得致密, 但出现较大尺寸的裂纹(裂纹宽度超过 $30 \mu \mathrm{m}$ ), 并 且伴随有碳纤维的䢃裂和缩颈现象 (图 4(f)、4(h))。 此外, 由于硅蒸汽与碳纤维的反应, 在碳纤维外表 面形成的 $\mathrm{SiC}$ 壳层也出现剥落的现象 (图 4(g)), 造成 这种现象是由于 $\mathrm{C} / \mathrm{C}$ 复合材料主要由碳纤维和碳基 体组成, 硅蒸汽与材料表面接触时, 首先在表面与 碳基体发生反应形成 $\mathrm{SiC}$ 层, 覆盖在材料的表面使 材料表面变得致密, $\mathrm{SiC}$ 与基底的热失配会产生较大 裂纹 $\left(\mathrm{C} / \mathrm{C}\right.$ 复合材料的热膨胀系数 $\left.1.0 \times 10^{-6} \mathrm{~K}^{-1}\right)$; 而对 于具有皮芯结构的碳纤维 ${ }^{[15]}$, 其与硅蒸汽的反应是 从外向里逐层反应, 外表面形成的 $\mathrm{SiC}$ 在热失配的 情况下逐渐剥落, 导致碳纤维在腐蚀过程中不断变 细, 出现 “缩颈” 现象。从图 4(i) (1)可以看出, 硬 质碳毡的内部有许多 $\mathrm{SiC}$ 纳米线的存在, 填充在材 料的孔隙中, 且其内部的碳纤维出现了明显碎断和 粉化(图 4(j)), 碳纤维表面的 $\mathrm{SiC}$ 壳层也有剥落现象, 并且出现类似“点蚀”的凹坑(图 4(1)), $\mathrm{s}$ 这是因为硬 质碳毡孔隙率较高, 硅蒸汽与碳纤维和碳基体反应 生成的 $\mathrm{SiC}$ 纳米线及颗粒无法在表面形成致密层, 而是渗入基体内部发生反应。另外, 由于制备碳毡
的纤维属低强度纤维 ${ }^{[16-17]}$, 反应后热失配应力远远 大于碳纤维自身的强度导致大量的碎断及粉化, 而 “点蚀坑”则主要是硅蒸汽优先与碳纤维表面的活性 点反应的不均匀腐蚀造成的。

\section{2 腐蚀参数对硅化腐蚀的影响}

图 5 为三种碳材料分别在 $1400 、 1500 、 1600^{\circ} \mathrm{C}$ 下 腐蚀 $5 \mathrm{~h}$ 后的表面微观形貌。随着腐蚀温度的升高, 石 墨表面整体致密度逐渐增加，但表面的微裂纹逐渐增 多, 裂纹宽度由 $0.5 \mu \mathrm{m}$ 逐渐增加到 $3 \mu \mathrm{m}$, 这些裂纹会 导致石墨材料力学性能的下降, 降低其使用寿命 ${ }^{[7]}$, $\mathrm{C} / \mathrm{C}$ 复合材料表面的致密度也逐渐增加，碳基体以及 碳纤维表面的 $\mathrm{SiC}$ 颗粒逐渐增多, 碳纤维与碳基体的 剥离现象也明显增加, 并且碳纤维表面的䢃裂现象越 来越严重，呈粉化趋势; 硬质碳段表面逐渐变得 “虚 化”, 内部纤维之间的缝隙被大量的 $\mathrm{SiC}$ 纳米线填充, 碳纤维表面的 $\mathrm{SiC}$ 颗粒逐渐增多, 纤维之间纳米线数 量增多, 碳纤维的碎裂现象越来越严重。

图 6 为不同温度下硅元素在材料中的扩散深度 (腐蚀时间均为 $5 \mathrm{~h}$ ), 由图可以看出, 三种碳材料由 于材料的致密度以及材料组成不一致，导致硅元 素的腐蚀深度有明显的区别: 石墨由于孔隙率较低, 且多为闭孔，其腐蚀深度最小; $\mathrm{C} / \mathrm{C}$ 复合材料内部有 较多的孔隙, 其腐蚀深度较石墨大; 而硬质碳段材 料由于内部孔隙率达到 $90 \%$, 孔隙相互联通, 给硅 蒸汽的扩散提供了通道, 其腐蚀深度远远大于石墨 和 $\mathrm{C} / \mathrm{C}$ 复合材料。另外, 石墨和 $\mathrm{C} / \mathrm{C}$ 复合材料中硅 元素的扩散深度随着温度的升高, 出现先升高后降 低的现象, 在 $1500^{\circ} \mathrm{C}$ 下达到最大值, 分别约为 $950 \mu \mathrm{m}$ 和 $960 \mu \mathrm{m}$; 而硬质碳毡材料中呈现明显的增加趋 势, 且硅元素的扩散深度远超过石墨和 $\mathrm{C} / \mathrm{C}$ 复合材 料, 当温度达到 $1600^{\circ} \mathrm{C}$ 时, 硅元素在硬质碳毡中 的扩散深度达到 $5 \mathrm{~mm}$, 超过测试材料厚度的一半, 


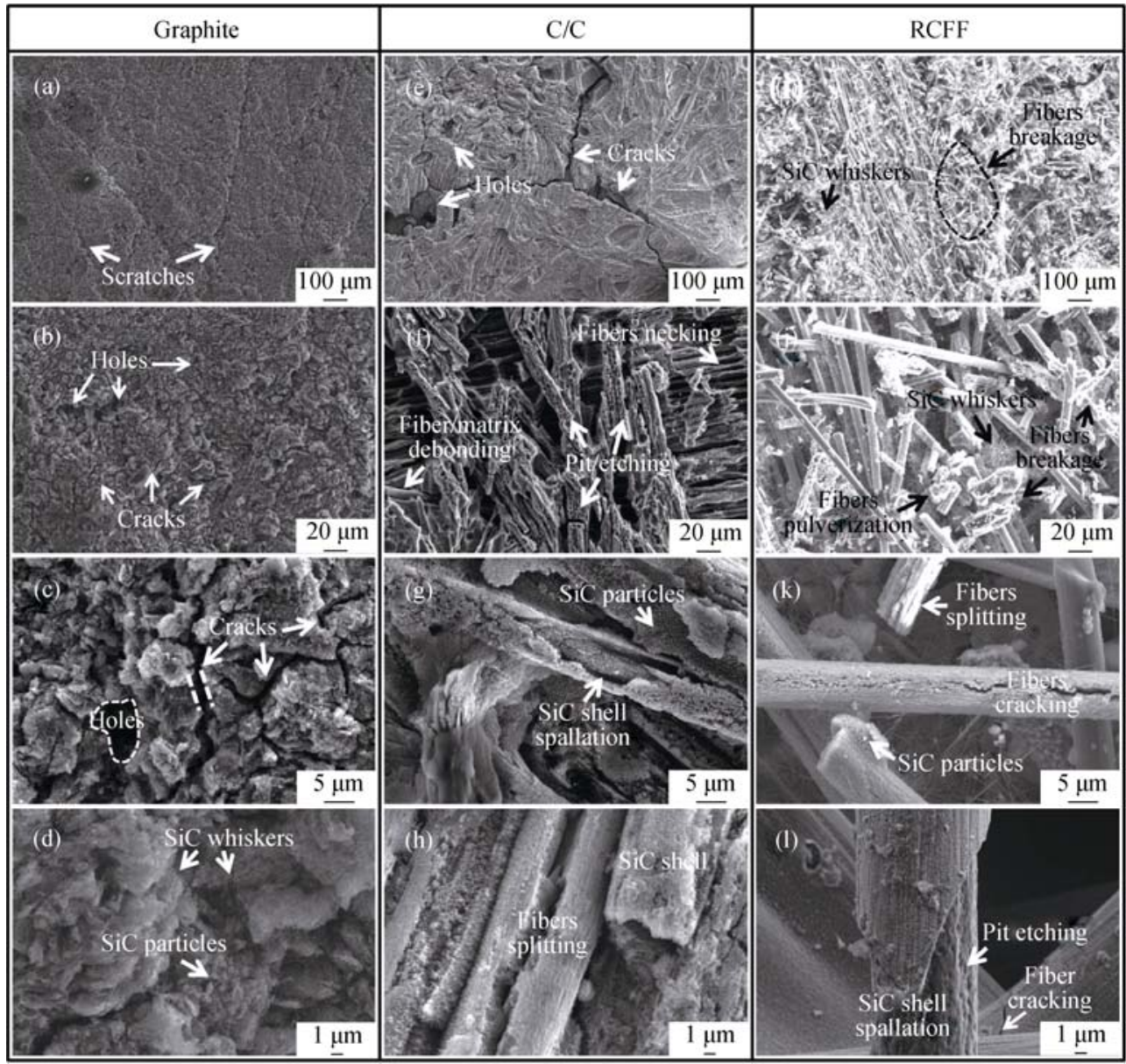

图 4 三种碳材料硅化腐蚀后的典型形貌特征

Fig. 4 Typical morphology characteristics of three carbon materials after silicification corrosion (a)-(d) Graphite; (e)-(h) C/C composites; (i)-(l) Rigid carbon fiber felt

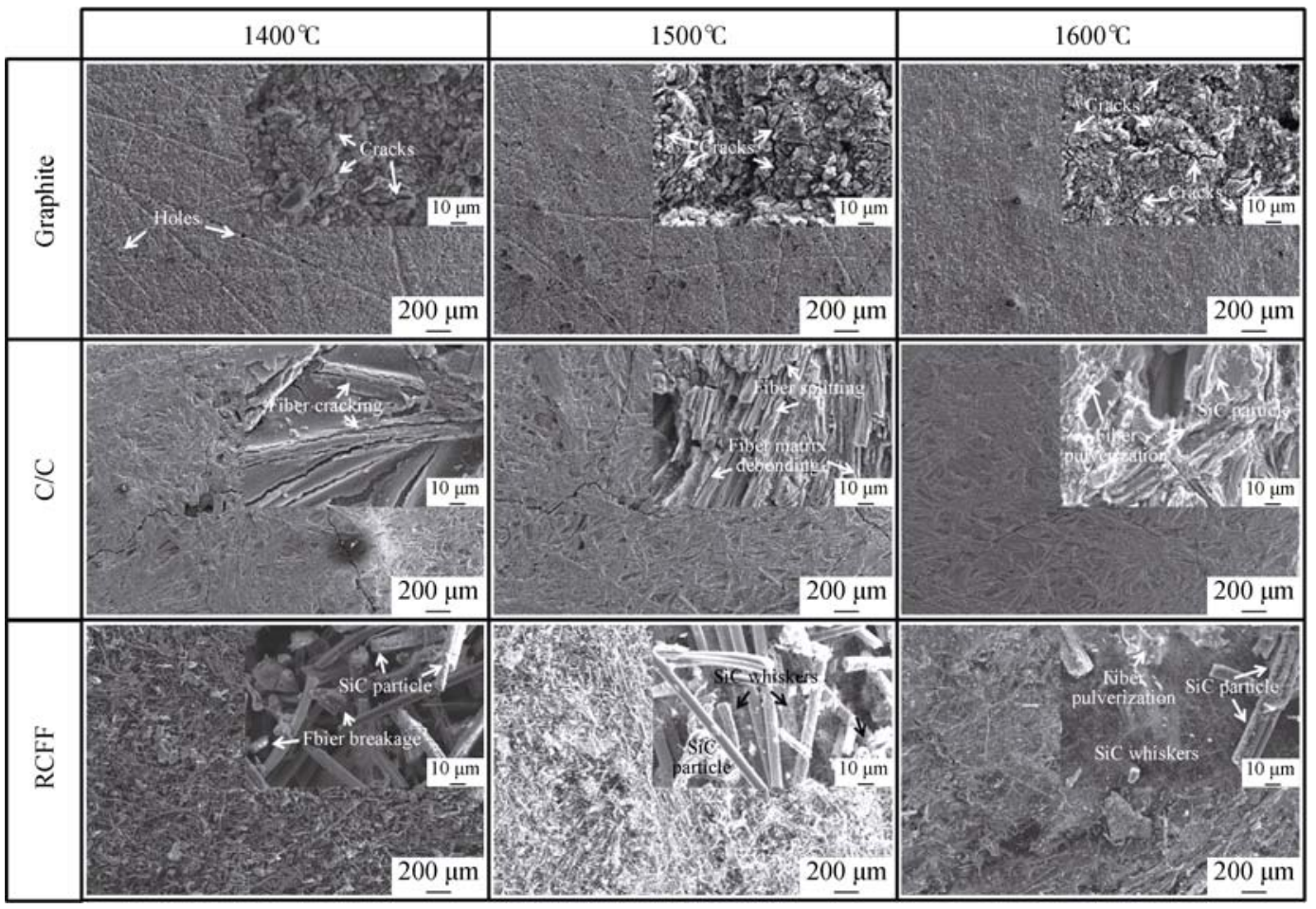

图 5 三种碳材料在不同温度下腐蚀后的微观形貌

Fig. 5 Microstructures of three carbon materials after corrosion at different temperatures 


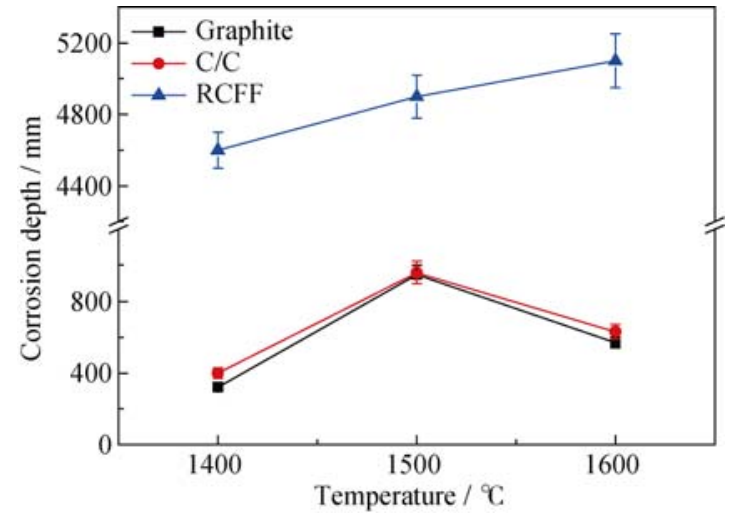

图 6 三种碳材料在不同温度下的腐蚀深度

Fig. 6 Corrosion depth of three carbon materials at different temperatures

腐蚀极其严重。

随着温度的升高, 硅的饱和蒸汽压逐渐增大 (如图 7 所示), 导致硅蒸汽的量增加, 且硅的扩散速 率也相应提高, 聚集在基底材料表面的反应气体浓 度也较高, 使得硅蒸汽与碳源充分接触, 加快了反 应速率，从而在基底碳材料表面迅速生成相对致密 的 $\mathrm{SiC}$ 层, 因此随着温度的升高, 材料表面的 $\mathrm{SiC}$ 层致密度逐渐增加。硅元素在碳材料内部的扩散主 要包括两种途径: 依靠材料孔隙和缺陷的气体扩散 过程和在材料内部的固体扩散过程。生成的致密
$\mathrm{SiC}$ 层在一定程度上会阻碍硅蒸汽向材料内部的扩 散, 使得硅元素的扩散逐渐由气体扩散变成固体扩 散, 虽然升高温度会促进硅元素在固体中的扩散速率, 但综合而言其扩散速率仍会大幅降低。在 $1600^{\circ} \mathrm{C}$ 腐蚀 条件下，由于温度较高，石墨以及 $\mathrm{C} / \mathrm{C}$ 复合材料表 面迅速生成一层致密的 $\mathrm{SiC}$ 层, 阻碍了硅蒸汽后续 的扩散, 因此降低了腐蚀深度; 而硬质碳毡由于孔 隙率高, 表面生成的 $\mathrm{SiC}$ 层无法形成致密层, 硅元 素在其内部的扩散始终由气体扩散主导，导致硅元 素的腐蚀深度持续增加。

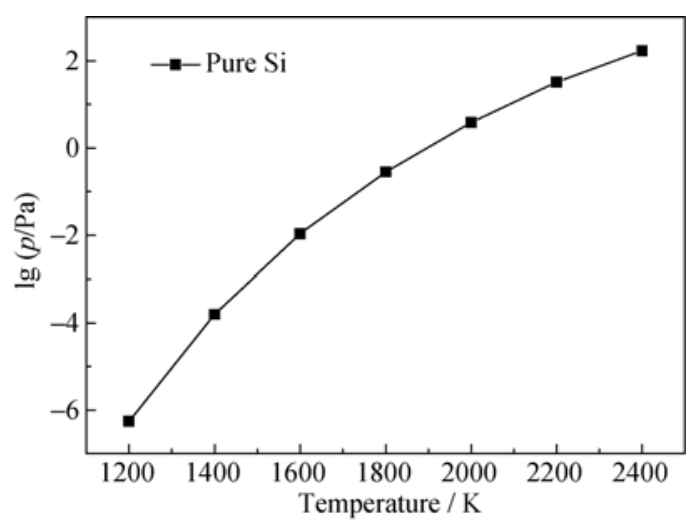

图 7 不同温度下硅的饱和蒸汽压曲线

Fig. 7 Saturation vapor pressure of silicon with different evaporation temperatures

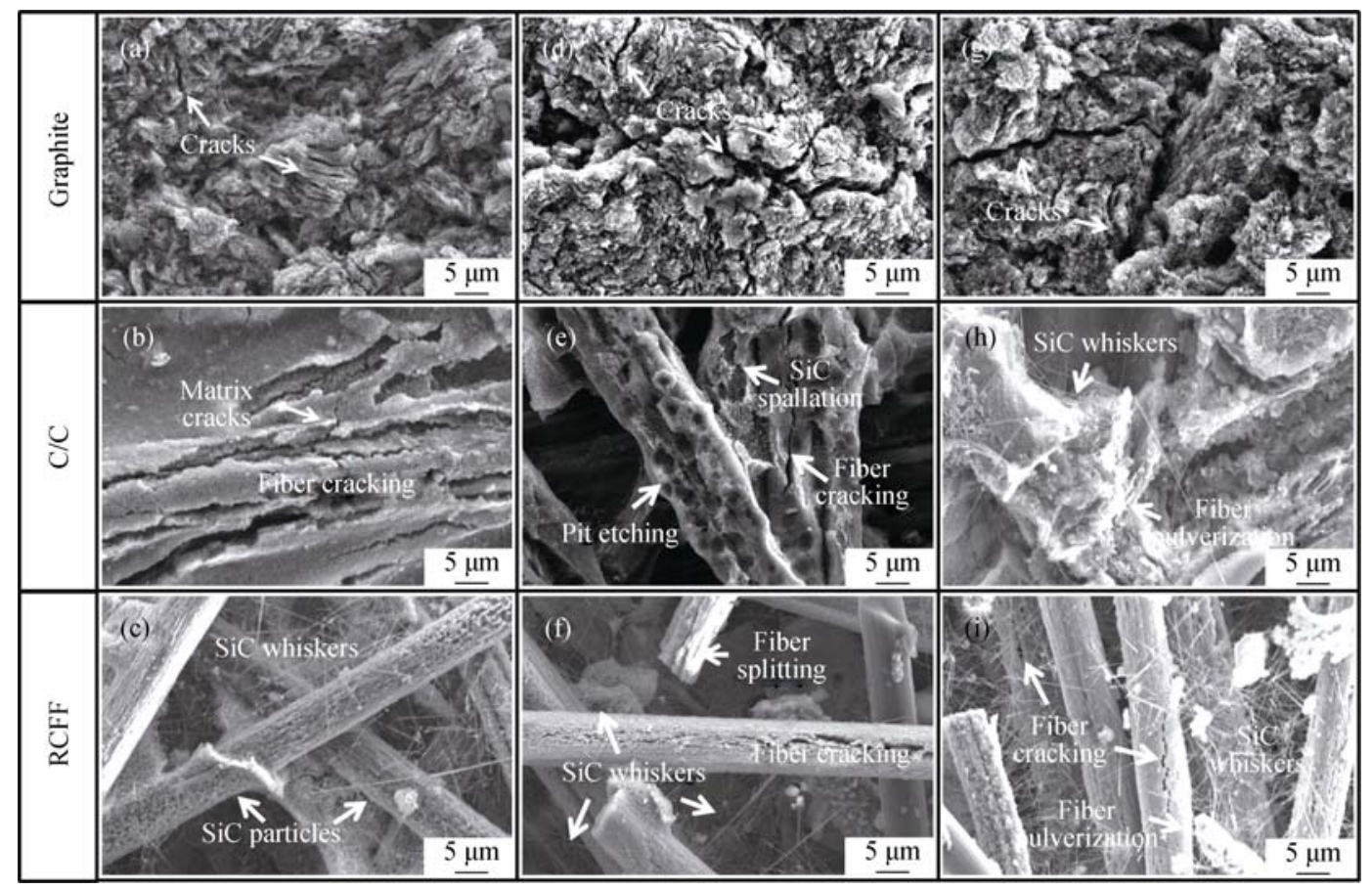

图 8 三种碳材料在 $1600^{\circ} \mathrm{C}$ 腐蚀不同时间的微观形貌

Fig. 8 Microstructures of three carbon materials corroded at $1600^{\circ} \mathrm{C}$ for different corrosion time (a)-(c) $1 \mathrm{~h}$; (d)-(f) $5 \mathrm{~h}$; (g)-(i) $10 \mathrm{~h}$ 
图 8 是三种碳材料在 $1600^{\circ} \mathrm{C}$ 下分别腐蚀 $1 、 5 、$ $10 \mathrm{~h}$ 后的微观形貌，从图中可以看出，随着硅化腐 蚀时间的延长，三种碳材料的宏观表面均变得致密， 但从微观上看石墨表面的裂纹增多, 裂纹宽度也增 大； $\mathrm{C} / \mathrm{C}$ 复合材料中碳基体和碳纤维的腐蚀程度明 显增加; 硬质碳钻中纤维的䢃裂和粉化现象也显著 增加，纤维间的 $\mathrm{SiC}$ 纳米线增多，上述现象基本与 腐蚀温度的影响类似。但是腐蚀时间对硅元素腐蚀 深度的影响却与温度的影响有所区别, 如图 9 所示, 三种碳材料的硅元素腐蚀深度随腐蚀时间的延长均 逐渐增加，但增加的趋势变得平缓。这是由于当腐 蚀温度一定, 硅的饱和蒸汽压以及扩散速率均相对 稳定，延长腐蚀时间有利于增加硅元素的扩散深度， 但是表面逐渐致密化的 $\mathrm{SiC}$ 层在腐蚀后期会阻碍 $\mathrm{Si}$ 元素的进一步渗入, 因此增加幅度趋于平缓。

\section{3 结论}

1）晶硅炉热场用的三种碳材料在高温环境下 均极易与硅蒸汽发生反应而产生腐蚀。硅化腐蚀后 碳材料的表面都会形成白色的、极易脱落的 $\mathrm{SiC}$ 纳 米线薄层。

2) 材料内部的硅化腐蚀主要是硅蒸汽通过材 料的孔洞以及缺陷扩散进入材料内部与碳材料反应 造成的。石墨材料内部存在的孔洞为气体的渗入提 供了通道, 反应生成的 $\mathrm{SiC}$ 造成石墨材料表面产生 大量微裂纹。 $\mathrm{C} / \mathrm{C}$ 复合材料和硬质碳毡材料中碳纤 维的存在方式以及纤维强度对硅化腐蚀程度有较大 影响, $\mathrm{C} / \mathrm{C}$ 复合材料中主要会产生碳基体和碳纤维 的剥离以及碳纤维的缩颈、䢃裂等; 而由强度较差 的短碳纤维为主要组成的硬质碳毡材料的腐蚀最严 重, 其内部碳纤维出现了明显的碎断、粉化。

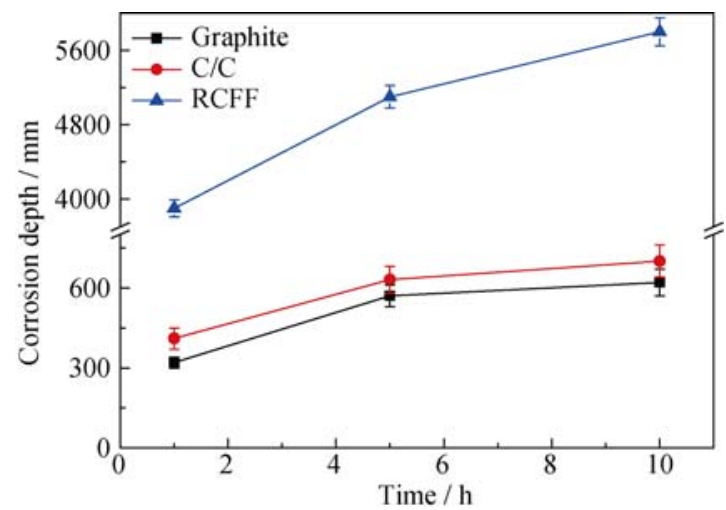

图 9 三种碳材料在 $1600^{\circ} \mathrm{C}$ 腐蚀不同时间的腐蚀深度

Fig. 9 Corrosion depth of three carbon materials corroded for different corrosion times
3) 随着腐蚀温度的升高, 三种材料的表面致 密度均有所增加，但是材料表面的微裂纹逐渐增多， 腐蚀程度显著提高; 石墨和 $\mathrm{C} / \mathrm{C}$ 复合材料的腐蚀深 度由于表面 $\mathrm{SiC}$ 晶粒的阻挡作用，呈现先增加后减 小的趋势; 而硬质碳段材料的表面孔隙过大，其腐 蚀深度持续增加，在 $1600^{\circ} \mathrm{C}$ 下腐蚀 $5 \mathrm{~h}$ 后的腐蚀深 度超过 $5 \mathrm{~mm}$ 。腐蚀时间对碳材料硅化腐蚀现象的影 响与腐蚀温度类似，但是三种材料的腐蚀深度均随 腐蚀时间的延长逐渐增加，增加幅度逐渐平缓。

\section{参考文献:}

[1] 孙 微, 贺 福. 太阳能光伏产业的热场材料. 高科技纤维与应 用, 2011, 36(1): 44-48.

[2] 彭志刚，肖志超，苏君明，等. 单晶硅拉制炉热场材料的发展概 况. 第 20 届炭・石墨材料学术会议, 成都, 2006: 204-207.

[3] 王世援, 韩焕鹏, 刘 锋. 碳/碳复合材料应用于直拉硅单晶生 长的研究. 电子工业专用设备, 2011, 195: 29-33.

[4] 陈青华, 侯卫权, 苏君明, 等. 多晶硅设备用炭/炭板材的研制. 第 21 届炭・石墨材料学术会议, 西安, 2008: 255-259.

[5] 梁仁和, 代红云, 侯英新. 多晶硅铸锭炉产业现状与发展前景. 新材料产业, 2011, 3: 28-30

[6] ZHAO W, ZHU B, CHEN Y, et al. The oxidation erosion in carbon materials for CZ furnace. Advanced Materials Research, 2013, 602-604(1662): 1359-1363.

[7] 曹伟伟, 朱 波, 赵 伟, 等. 单晶硅炉用碳素材料的硅化腐蚀研 究. 功能材料, 2013, 44(12): 1709-1712.

[8] ZHOU X W, LU Z M, LI X N, et al. The oxidation behavior of A3-3 matrix graphite. New Carbon Materials, 2016, 31(2): 182-187.

[9] 陈志军, 奚静伦. 碳/碳复合材料的氧化性能研究. 航空工艺技 术, 1990, 4(5): 12-16.

[10] 徐 伟, 石 否, 郑艳华, 等. 孔隙率对核级石墨氧化的影响. 原 子能科学技术, 2016, 50(3): 471-476.

[11] 廖寄乔, 黄伯云, 石 刚, 等. C/C 复合材料开孔孔隙度和比表面 积对其氧化性能的影响. 中南工业大学学报，2002，33(5): 500-504.

[12] Thermfact/CRCT and GTT Technologies, http://www.factsage. com/.

[13] 吴玲玲, 吴仁兵, 杨光义，等. 硅热蒸发法制备 $\mathrm{SiC}$ 纳米线 及其结构表征. 浙江工业大学学报(工学版)，2008，42(3): 485-488

[14] DAI J X, SHA J J, ZHANG Z F, et al. Synthesis of high crystalline beta $\mathrm{SiC}$ nanowires on a large scale without catalyst. Ceramics International, 2015, 41(8): 9637-9641.

[15] LIU X F, ZHU C Z, GUO J, et al. Nanoscale dynamic mechanical imaging of the skin-core difference: From PAN precursors to carbon fibers. Materials Letters, 2014, 128: 417-420.

[16] SHI W, LI J Y, YOU Q F, et al. Preparation and properties of rigid carbon felt thermal insulation. Materials Science Forum, 2015, 833(1662): 48-51.

[17] SHI W, TAN Y, YOU Q F, et al. Erosion resistant composite coating on rigid carbon fiber felt. Ceramics International, 2016, 42(5): $5823-5829$. 